\title{
Electrostatic coupling driven dielectric enhancement of PZT/BTO multilayer thin films
}

\section{Huang Zhong}

South China University of Technology

Xin Wang ( $\sim$ g96217@scut.edu.cn )

South China University of Technology https://orcid.org/0000-0002-6646-8795

\section{Xin Hong Li}

South China University of Technology

\section{Zhen Ya Lu}

South China University of Technology

\section{Zhi Wu Chen}

South China University of Technology

\section{Research Article}

Keywords: sol-gel, dielectric enhancement, PZT/BTO, internal field

Posted Date: June 7th, 2021

DOl: https://doi.org/10.21203/rs.3.rs-267404/v1

License: (c) (i) This work is licensed under a Creative Commons Attribution 4.0 International License. Read Full License 


\section{Title Page:}

·Huang Zhong, Xin Wang *, Xinhong Li, Zhenya Lu, Zhiwu Chen

-Electrostatic coupling driven dielectric enhancement of PZT/BTO multilayer thin films

-School of Materials Science and Engineering, South China University of Technology, Guangzhou 510640, China

·Xin Wang*Ｅ-mail: g96217@scut.edu.cn

-ORCID:0000-0002-8322-3546

\section{Abstract}

In this study, $\mathrm{PbZr}_{0.52} \mathrm{Ti}_{0.48} \mathrm{O}_{3} / \mathrm{BaTiO}_{3}$ ( $\mathrm{PZT} / \mathrm{BTO}$ ) multilayers with varying layer fractions were deposited on $\mathrm{Pt} / \mathrm{Ti} / \mathrm{SiO}_{2} / \mathrm{Si}$ substrates by sol-gel process. The films were characterized by $\mathrm{X}$-ray diffraction and scanning electron microscopy (SEM). The result shows that there exists a dielectric enhancement when the BTO layer fraction $x$ is around 0.5 , and at this fraction, the dielectric constant of PZT/BTO is 590 at $100 \mathrm{kHz}$, which is far more than that of monolithic PZT or BTO films (478 and 284, respectively). The thermodynamic analysis shows that the measured dielectric constant is close to the simulation values when $\mathrm{x}$ closes to 0.5 , otherwise it better consists with the series connection calculation values. The result indicates that the internal field resulting from the polarization mismatch between two ferroelectric layers contributes to the enhancement of PZT/BTO heterogeneous thin films.

Keywords: sol-gel, dielectric enhancement, PZT/BTO, internal field

\section{Declarations :}

Funding: This work was financially supported by National Natural Science Foundation of China (U1601208 and 51577070), the Key-Area Research and Development Program of Guangdong Province (2019B010937001, 2019B040403004, 2019B040403006), Natural Science Foundation of Guangdong Province (2019A1515012129), and Science and Technology Planning Project of Guangzhou City (202002030420).

Conflict of interest: The authors have no conflicts of interest to declare that are relevant to the content of this article.

\section{Relevance Summary}

The electrostatically driven dielectric enhancement behaviors of PZT/BTO multilayer thin films with varying layer fractions onto $\mathrm{Pt} / \mathrm{Ti} / \mathrm{SiO}_{2} / \mathrm{Si}$ substrate were investigated. Better crystallinity and surface topography were found of heterogeneous films. For the better interlayer coupling effect, there exist a dielectric and ferroelectric enhancement when the BTO layer fraction $\mathrm{x}$ is around 0.5.the dielectric properties of PZT/BTO are closer to the max theoretical values calculated by thermodynamic model.

This work was carried out with many research and application values. First, it improved the deterioration of electrical properties caused by thinning PZT thin films. Second, the principle of dielectric enhancement of PZT-based Ferroelectricity-Ferroelectricity bilayer ferroelectric films were studied here. It means that we can make use of the sol-gel or other simple industry method to obtain unique dielectric responses as many other complicated works have done. For a miniature integrated capacitor with limited dimensions, this method enables the capacitor to have more storage capability while maintain relatively low industrial costs. 


\title{
Electrostatic coupling driven dielectric enhancement of PZT/BTO multilayer thin films
}

\author{
Huang Zhong, Xin Wang *, Xinhong Li, Zhenya Lu, Zhiwu Chen \\ (School of Materials Science and Engineering, South China University of Technology, Guangzhou \\ 510640, China)
}

\begin{abstract}
In this study, $\mathrm{PbZr}_{0.52} \mathrm{Ti}_{0.48} \mathrm{O}_{3} / \mathrm{BaTiO}_{3}$ ( $\mathrm{PZT} / \mathrm{BTO}$ ) multilayers with varying layer fractions were deposited on $\mathrm{Pt} / \mathrm{Ti} / \mathrm{SiO}_{2} / \mathrm{Si}$ substrates by sol-gel process. The films were characterized by $\mathrm{X}$-ray diffraction and scanning electron microscopy (SEM). The result shows that there exists a dielectric enhancement when the BTO layer fraction $x$ is around 0.5 , and at this fraction, the dielectric constant of PZT/BTO is 590 at $100 \mathrm{kHz}$, which is far more than that of monolithic PZT or BTO films (478 and 284, respectively). The thermodynamic analysis shows that the measured dielectric constant is close to the simulation values. The result indicates that the internal field resulting from the polarization mismatch between two ferroelectric layers contributes to the enhancement of PZT/BTO heterogeneous thin films.
\end{abstract}

Keywords: sol-gel, dielectric enhancement, PZT/BTO, internal field

\section{Introduction}

PZT and BTO films have been widely studied because of their excellent properties like ferroelectric, dielectric, pyroelectricity and so on. They have been used in many devices like nonvolatile ferroelectric random access memories (NVFRAM) [1], microelectromechanical systems (MEMS) [2], capacitors [3] and some detectors [4]. However, high density and high integration electronic products require further reduction of capacitor size and increase the specific capacitance, the film thickness will be declined to meet the requirement, and this is accompanied by the deterioration of dielectric properties, film morphology and flatness [5,6]. Therefore, the multilayer structure films were studied to solve the deterioration of monolithic films because of the unique properties that they exhibit $[7,8]$. The early studies about the multilayers structure like ferroelectric (FE)-ferroelectric (FE) and ferroelectric (FE)paraelectric (PE) suggest that there is a strong inter-layer coupling that must be considered to account for the properties of FE heterostructures $[9,10]$, which contribute to the anomaly of electrical properties.

Although there are various explanations for the interlayer interaction of multilayer ferroelectric films like phase field model and Ising-type of computations [11,12], the most recognized one is the interlayer electrostatic coupling effect based on thermodynamic modeling [13]. Because of the polarization mismatch [14] between the layers, there exists a built-in electric field between FE-FE or FEPE bilayers, and its electric field strength may change with different layer properties and fraction. It may affect the polarization response of multilayer films, and lead to dielectric anomalies at critical layer fraction. Based on this, Ruguan Li's experiment demonstrates that the built-in electric field at the region near the $\mathrm{Bi}_{1.5} \mathrm{Zn}_{1.0} \mathrm{Nb}_{1.5} \mathrm{O}_{7} / \mathrm{Ba}_{0.5} \mathrm{Sr}_{0.5} \mathrm{TiO}_{3}$ (BZN/BST) interface is responsible for the asymmetric characteristic of the electric-field-dependent dielectric properties of the BZN/BST thin films [15]. $\mathrm{Ca}\left(\mathrm{Mg}_{1 / 3} \mathrm{Nb}_{2 / 3}\right) \mathrm{O}_{3} / \mathrm{CaTiO}_{3}(\mathrm{CMN} / \mathrm{CT})$ [16] heterogeneous films had been deposited on Pt electrode with different layer thickness. The depletion layer at the interface helps improve surface morphology and the dielectric properties, the $\mathrm{CMN} / \mathrm{CT}$ heterogeneous films exhibit the excellent dielectric properties of $\varepsilon_{r}=$ $67.7, \tan \delta=1.36 \%$ at the frequency of $1 \mathrm{MHz}$. Khassaf. $\mathrm{H}$ [17] found that there exist ferroelectricity 
vanishes in $\mathrm{PbZr}_{0.2} \mathrm{Ti}_{0.8} \mathrm{O}_{3} / \mathrm{SrTiO}_{3}(\mathrm{PZT} / \mathrm{STO})$ heterogeneous films when the ratio of STO is less than $30 \%$, associated with the disappearance of ferroelectric behavior, he assumes that there exists a significant increase of dielectric constant because of the internal fields between the two layers. Similar studies have been carried on many other FE based heterogeneous thin films [18-22]. For example, the $\mathrm{Pb}\left(\mathrm{Zr}_{0.8} \mathrm{Ti}_{0.2}\right) \mathrm{O}_{3} / \mathrm{Pb}\left(\mathrm{Zr}_{0.2} / \mathrm{Ti}_{0.8}\right) \mathrm{O}_{3}$ structure's electrical properties can be enhanced at an optimal value when the bilayers at a critical layer fraction with sharply enhanced $P_{r}$ of $32.6 \mu \mathrm{C} / \mathrm{cm}^{2}$ [23].

However, there are few systematic studies about the dielectric anomaly of PZT/BTO heterogeneous films, such an investigation will be helpful for the dielectric tunability of PZT based heterogeneous films. In this study, $\mathrm{PbZr}_{0.52} \mathrm{Ti}_{0.48} \mathrm{O}_{3} / \mathrm{BaTiO}_{3}\left(\mathrm{PZT} / \mathrm{BTO}\right.$ ) heterogeneous thin films on $\mathrm{Pt} / \mathrm{Ti} / \mathrm{SiO}_{2} / \mathrm{Si}$ substrates were deposited by sol-gel method. Both PZT and BTO have good dielectric and ferroelectric properties. The phase structure, microstructure, dielectric and ferroelectric properties were reported and analyzed in detail. The anomaly of PZT/BTO is explained by thermodynamic modeling, and it has been compared with series-parallel structure to further instruct the electrostatic coupling between films.

\section{Experimental}

\subsection{Fabrication of multilayers}

The PZT/BTO multilayer thin films were fabricated by sol-gel method. Lead acetate trihydrate $\left[\mathrm{Pb}\left(\mathrm{CH}_{3} \mathrm{COO}\right)_{2}\right]$, Zirconyl nitrate $\left[\mathrm{ZrO}\left(\mathrm{NO}_{3}\right)\right]$, Titanium butoxide $\left[\mathrm{C}_{16} \mathrm{H}_{36} \mathrm{O}_{4} \mathrm{Ti}\right]$, Barium acetate $\left[\mathrm{Ba}\left(\mathrm{CH}_{3} \mathrm{COO}\right)_{2}\right]$, Acetylacetone, 2-Methoxyethanol and Acetic acid were used as raw materials for PZT and BTO precursor solution. 10\% excess lead is added to account for the volatilization of lead during heat treatment. Both PZT and BTO solution's concentration is $0.3 \mathrm{~mol} / \mathrm{L}$, and the $\mathrm{pH}$ was controlled at 4. After 24 hours of storage, the precursors were spun onto $\mathrm{Pt} / \mathrm{Ti} / \mathrm{SiO}_{2} / \mathrm{Si}$ substrates $(1 \mathrm{~cm} \times 1 \mathrm{~cm})$ at different rotation rate to obtain different film thicknesses. The first layer was dried at $120^{\circ} \mathrm{C}$ for $3 \mathrm{~min}$, followed by pyrolysis of the organic at $350{ }^{\circ} \mathrm{C}$ for $15 \mathrm{~min}$, and then the film was annealed at $600{ }^{\circ} \mathrm{C}$ for $10 \mathrm{~min}$. After the first layer was prepared, the second layer was spun and heat treated by the same way. Finally, the PZT/BTO was annealed at $720{ }^{\circ} \mathrm{C}$ for $30 \mathrm{~min}$ by rapid thermal annealing (RTA). In order to form the MIM structure, the corner of the film was etched off with hydrofluoric acid (HF), and $\mathrm{Au}$ electrodes with a diameter of $0.5 \mathrm{~mm}$ were then deposited on the film surface by magnetron sputtering. The total thickness $h$ was controlled at about $300 \mathrm{~nm}$, and the BTO layer thickness fraction $x$ is defined as $x=h_{B T O} /\left(h_{P Z T}+h_{B T O}\right)$. The schematic of PZT/BTO heterogeneous films is shown in Figure 1.

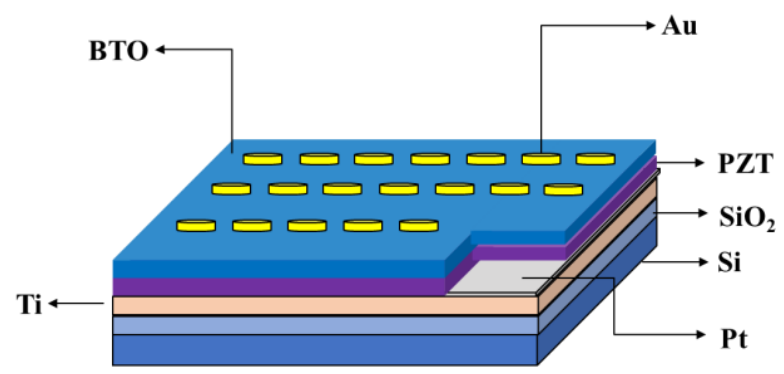

Fig.1 The schematic of the structure of PZT/BTO heterogeneous films

\subsection{Characterization}

The crystal structure was determined by X-ray diffraction (X'Pert Pro, PANalytical, Netherlands) with $\mathrm{Cu} \mathrm{K \alpha}$ radiation $(\lambda=1.5406 \AA)$. The surface morphology and the cross-section were observed by scanning electron microscope (SEM) (Merlin, Zeiss, Germany). The dielectric properties were examined by precision impedance analyzer (4294A, Agilent, US) from $100 \mathrm{~Hz}$ to100 kHz. The leakage current 
measurements were characterized by High Voltage Source Meter (2410, Keithley, US) from 0 to 120 $\mathrm{kV} / \mathrm{cm}$. The P-E hysteresis loops were characterized by a modified Sawyer-Tower circuit (Radiant Precision Multiferroic, DEI Technology, US). All the measurements were taken at room temperature.

\section{Results and discussion}

\subsection{Phase structure and morphology of PZT/BTO thin films}
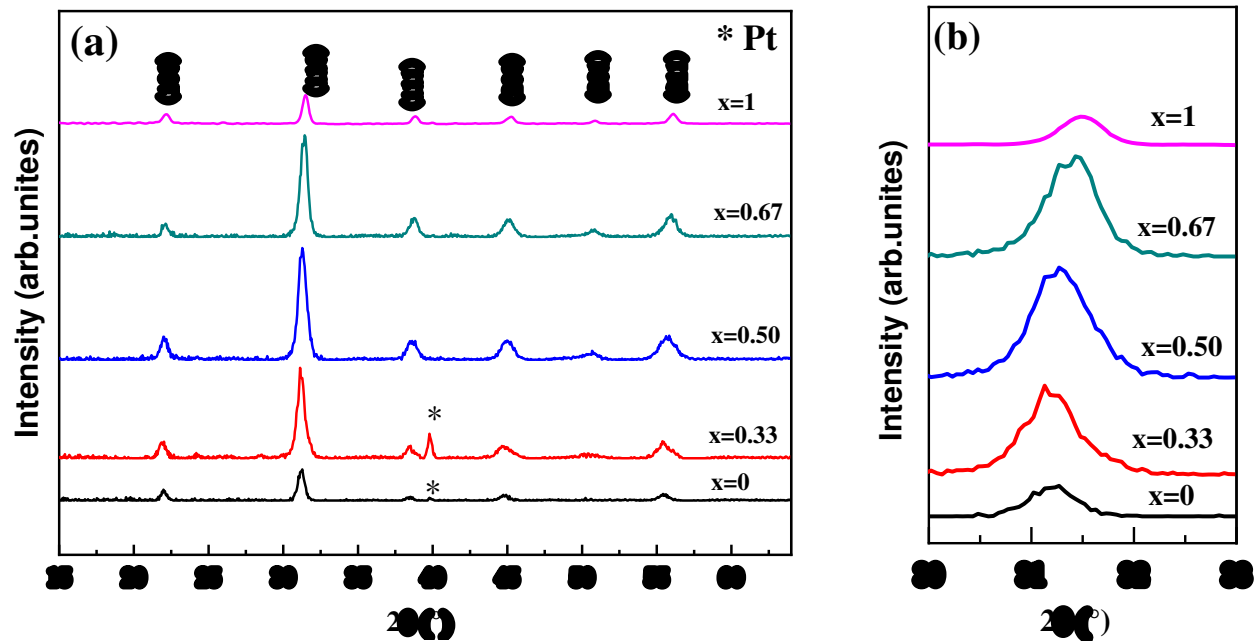

Fig.2 XRD patterns of PZT/BTO heterogeneous films with different $x$ values (a), locally enlarged view of the (110) peak (b)

Table 1. The average lattice parameters of PZT/BTO multilayer films

\begin{tabular}{cccccc}
\hline$x$ & 0 & 0.33 & 0.5 & 0.67 & 1 \\
\hline PZT & $0.404 \mathrm{~nm}$ & $0.406 \mathrm{~nm}$ & $0.404 \mathrm{~nm}$ & $0.402 \mathrm{~nm}$ & $/$ \\
BTO & $/$ & $0.404 \mathrm{~nm}$ & $0.405 \mathrm{~nm}$ & $0.403 \mathrm{~nm}$ & $0.402 \mathrm{~nm}$ \\
\hline
\end{tabular}

Fig. 2(a) shows the XRD patterns of the PZT/BTO multilayer thin films of different $x$ ratio, and there is an inconspicuous split peak in (110) as depicted in Fig. 2(b). It can be discovered from the pattern of $x=0$ and $x=1$ that the PZT and BTO were both pure and polycrystalline. No impurity phases can be detected in the PZT/BTO bilayer films. which means there didn't form a solid solution but the single phase in their own layer. The peaks of the heterogeneous bilayer film $(x=0.33,0.50,0.67)$ are significantly higher than the monolithic one $(x=0,1)$, it has been demonstrated that a slight lattice mismatch between two layers can reduce the lattice relaxation and promote the crystallization [24], 2D layer-by-layer growth mode can be maintained. According to diffraction formula $2 d \sin \theta=n \lambda$, the average lattice parameter of PZT and BTO are shown in table 1, both PZT and BTO's lattice parameter decrease with increasing $x$, and a slight shift to the higher angles of the diffraction peaks was also observed. Even no second phase can be detected, formation of intermediate layers due to the interfacial diffusion between the PZT and BTO layers during the process of heat treatment, leading to the reduction of lattice parameter. However, because the pre-crystallization of the first layer was well grown before the second layer was deposited, the elemental diffusion is not obvious, which leading to a slightly shift of the diffraction peaks. Therefore, there is a well-defined multilayer film as the XRD result shows. 

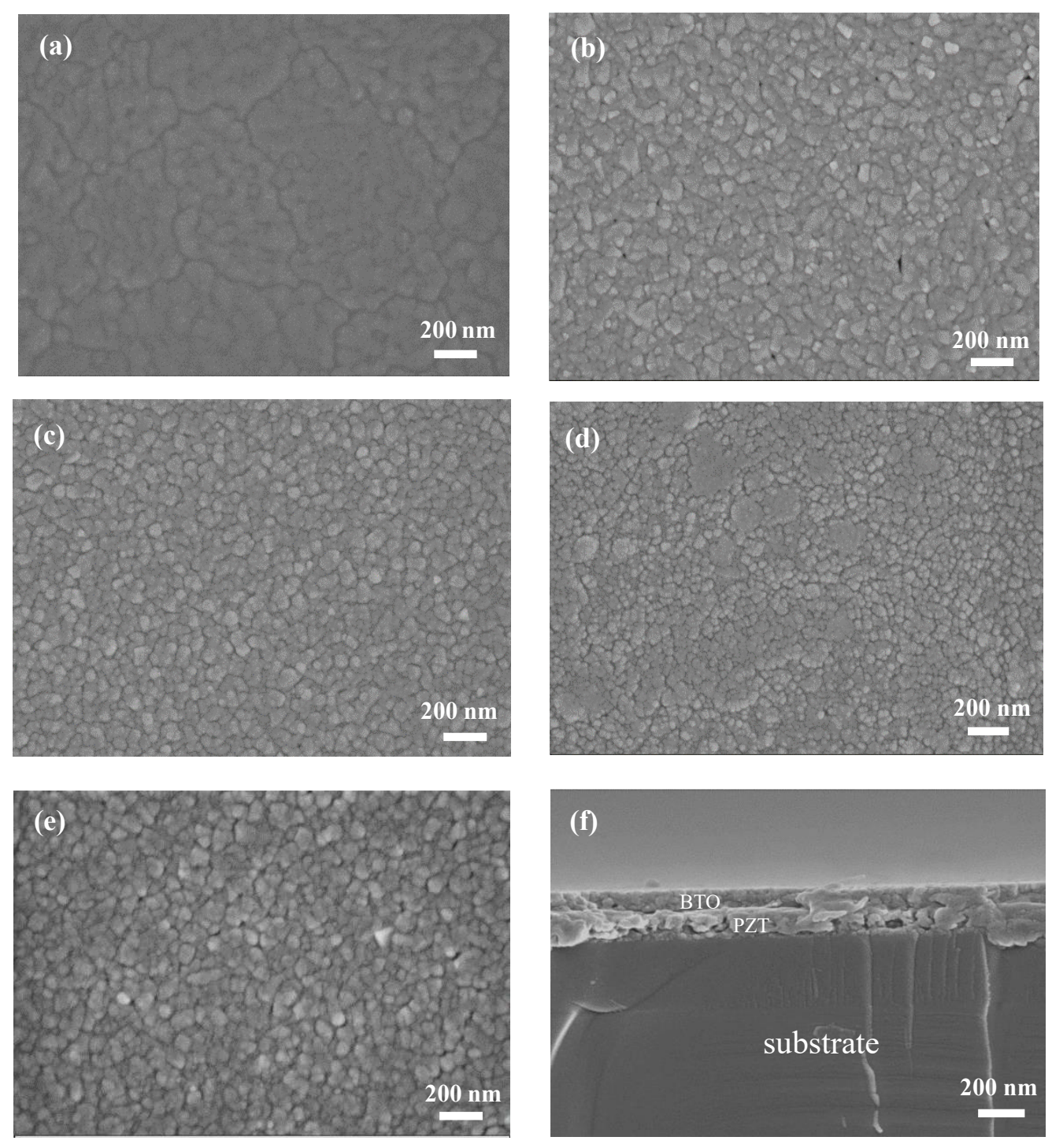

Fig.3 SEM images of the PZT/BTO surface of different $\mathrm{x}$ ratio: $x=0(\mathbf{a}), x=0.33$ (b), $x=0.50$ (c), $x=0.67$ (d), $x$ $=1$ (e), the cross-section view of $x=0.50$ (f)

Fig. 3(a)-(e) are SEM images of sample $x=0,0.33,0.50,0.67,1.00$, respectively. It can be easily discovered that the heterogeneous structure has smaller grain size and smoother surface than monolithic PZT or BTO film, the density of the PZT/BTO thin films has been improved, too. Fig.3(c) $(x=0.50)$ shows the best uniformity and flatness. Just as analyzed in the XRD patterns, the lattice mismatch between two heterogeneous layers contributed to reduce the lattice relaxation and promoted the crystallization. What's more, it has been reported that excess compressive stresses cause the quasihomogeneous phase boundary of PZT to move in the Zr-rich direction [25], which is detrimental to the stabilization of monoclinic phases in quasi-homogeneous PZT films. When the BTO component increases, the compressive stress on the PZT resulted in the decreases of the overall grain size, this change also affected its ferroelectric properties in the later discussion. Fig.3(f) presents the SEM image of a cross-sectional heterogeneous film on $\mathrm{Pt} / \mathrm{Ti} / \mathrm{SiO}_{2} / \mathrm{Si}$ substrate when $x=0.5$, it shows dense and uniform microstructures across the film thickness.

\subsection{Dielectric properties of PZT/BTO heterogeneous thin films}




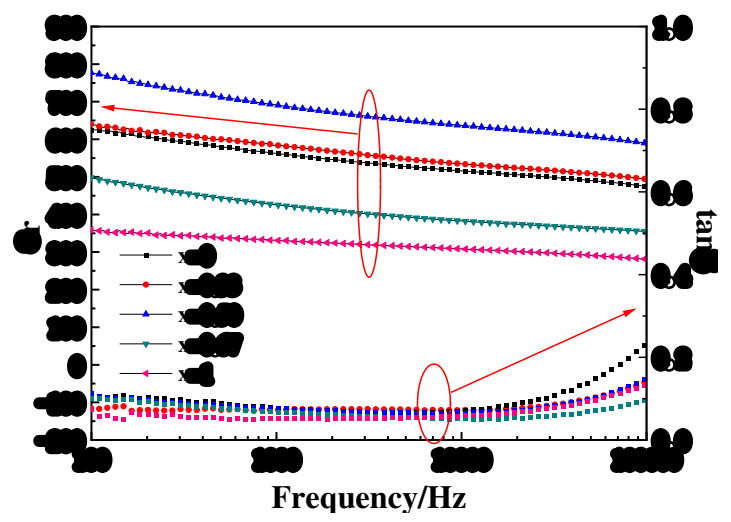

Fig.4 Dielectric constant and loss tangent of PZT/BTO heterogeneous thin films

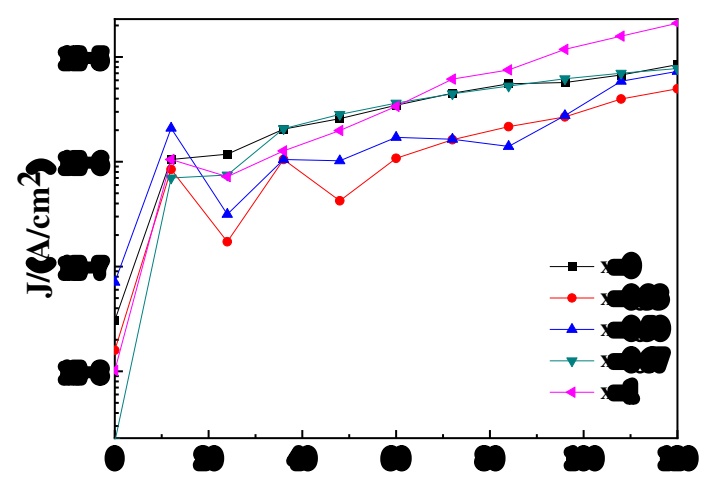

Electric field, $\mathrm{E}(\mathrm{kV} / \mathrm{cm})$

Fig.5 Leakage current density of PZT/BTO heterogeneous thin films

The dielectric constant $\varepsilon_{r}$ and loss tangent $\tan \delta$ as a function of frequency for the PZT/BTO heterogeneous thin films is shown in Fig.4. It indicates that the dielectric constant in the range of $100 \mathrm{~Hz}$ to $100 \mathrm{kHz}$ shows an overall downward trend with the increasing of the frequency. However, at the same frequency, the dielectric constant grows with the increase of $\mathrm{x}$, when $x=0.5$, the dielectric constant of PZT/BTO reaches the maximum value, which is 590 at $100 \mathrm{kHz}$. Then it decreases with the increasement of $x$. This may be related to the electrostatic coupling between thin film layers, it will be discussed in detail in later section. It can be found that, at $100 \mathrm{kHz}$ when $x=0.33,0.50,0.67$, the loss tangent is 0.143 , $0.150,0.099$, respectively. However, the loss tangent is 0.236 and 0.145 when $x=0$ and 1 , which means that the heterogeneous thin films have lower loss tangent than monolithic PZT or BTO films. The leakage current density of PZT/BTO, as it was shown in Fig.5, further confirms this, when $x=0$ or 1 , the monolithic PZT and BTO have higher leakage current density than that $x=0.33,0.50,0.67$. The results are consistent with the XRD analysis and the surface topography, the better crystallization and uniform microstructure help reduce loss tangent during operation. There are two reasons for a lower leakage current density. The first one is due to the formation of transition layer between PZT and BTO, the transmission of electrons becomes more difficult. The second reason is that the inter-layer mismatch between PZT and BTO allows for better film uniformity, changing from island stacking to staggered stacking structure [26].

The well-defined hysteresis loops of the PZT/BTO heterogeneous thin films of different $x$ ratio are depicted in Fig.6(a). All PZT/BTO films of different $x$ ratio exhibit varying degrees of slanted hysteresis loop. The monolithic PZT and BTO were both ferroelectric materials, their combination also has the properties of ferroelectrics. Fig.6(b) and Fig.6(c) show the remanent polarization $\left(P_{r}\right)$ and coercive field $\left(E_{c}\right)$ as a function of applied voltage. It can be discovered that the $P_{r}$ has almost the same pattern of variation to the phenomenon of the dielectric constant analyzed earlier. According to the MaxwellWagner effect [27,28], the space charge polarization plays an important part in the heterogeneous films and it contributes to the total polarization. Besides, the coercivity field of the film becomes higher when the $x$ is equal to or greater than 0.67 . Therefore, it can be indicated that when $x$ is close to 0.5 , the $\mathrm{PZT} / \mathrm{BTO}$ heterogeneous thin film has the best ferroelectric properties. 

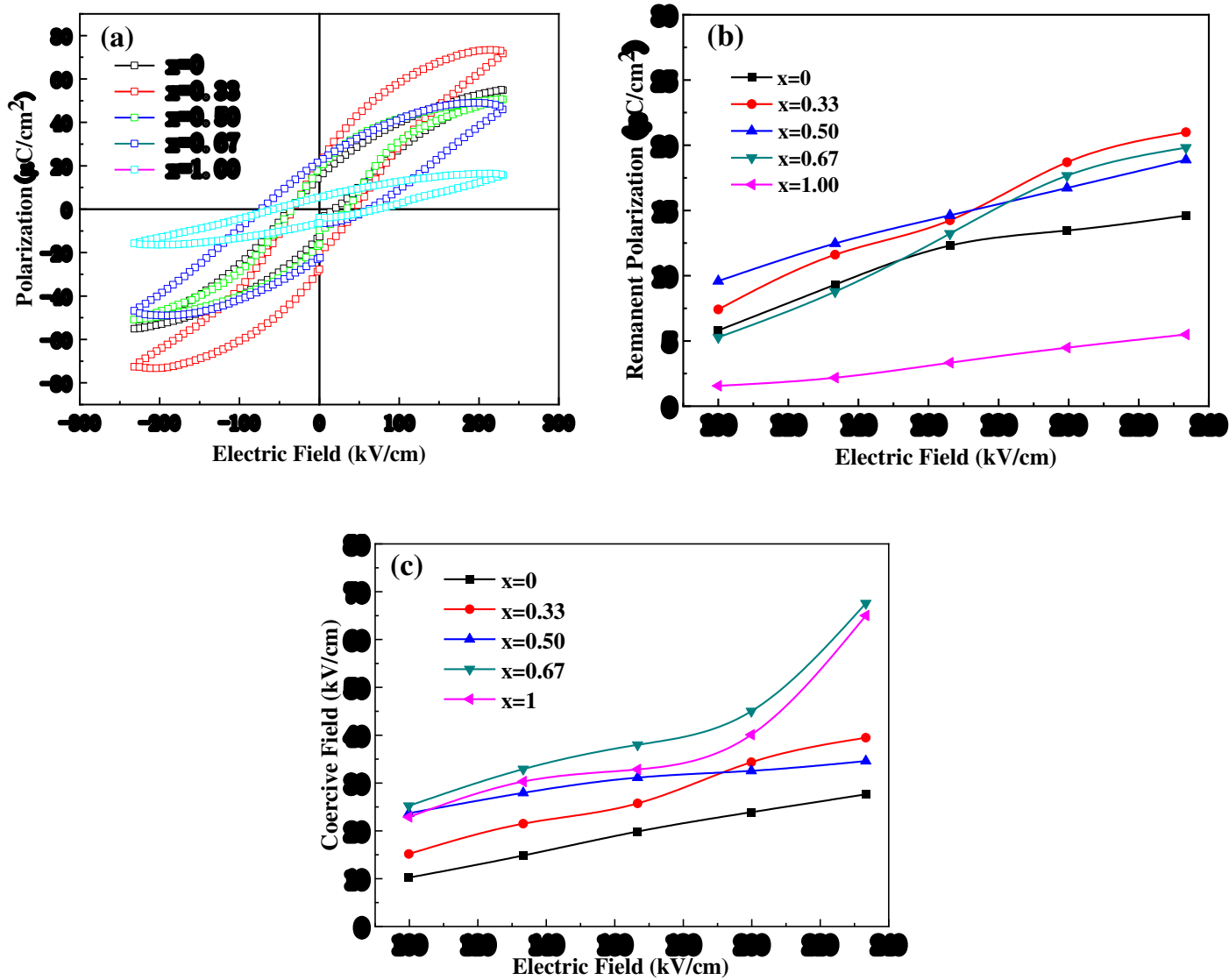

Fig.6 PZT/BTO heterogeneous films with different thickness ratios of: the hysteresis loop (a), remanent polarization $\left(P_{r}\right) \mathbf{( b )}$, coercive field as a function of applied field $\left(E_{c}\right)(\mathbf{c})$

\subsection{Theoretical analysis and discussion}

A thermodynamic model was employed here to explain the dielectric anomaly of PZT/BTO heterogeneous films. Assuming that the thickness of the substrate is much greater than the thickness of the film, so that all internal stresses are concentrated in the film, for monolithic ferroelectric films, the free energy of the bilayer can be presented as [29]:

$$
G\left(P_{i}\right)=G_{0, i}+\frac{1}{2} \tilde{a}_{i} P_{i}^{2}+\frac{1}{4} \tilde{b}_{i} P_{i}^{4}+\frac{1}{6} c_{i} P_{i}^{6}
$$

where the $\mathrm{P}$ is the polarization, $\tilde{a}_{i}$ and $\tilde{b}_{i}$ are renormalized dielectric stiffness coefficients, which is related to mismatch between film and substrate [30]. $c_{i}$ is the bulk dielectric stiffness coefficient, the details are given by elsewhere [29,31]. For PZT/BTO heterogeneous films, in addition to the proportions of the film itself and its interaction with the substrate, the effect of electrostatic coupling between the layers should also be considered [32]:

$$
G=(1-x)\left[G_{1}\left(P_{1}\right)-E P_{1}\right]+x\left[G_{2}\left(P_{2}\right)-E P_{2}\right]+\frac{x(1-x)}{2 \varepsilon_{0}}\left(P_{1}-P_{2}\right)^{2}
$$

where the $\mathrm{E}$ is an applied electrical field parallel to the polarization, and $\varepsilon_{0}$ is the vacuum dielectric constant. The polarization equation for each layer can be calculated by equilibrium $\frac{\partial G}{\partial P_{1}}=0$ and $\frac{\partial G}{\partial P_{2}}=$ 0 . The average dielectric response of the heterogeneous film is: 


$$
\varepsilon_{r}=\frac{1}{\varepsilon_{0}} \frac{d P}{d E}
$$

where $\mathrm{P}$ presents the average polarization:

$$
P=(1-x) P_{1}+x P_{2}
$$

and thus equation (3) can be described as

$$
\varepsilon_{r}=\frac{1}{\varepsilon_{0}}\left[(1-x) \frac{d P_{1}}{d E}+x \frac{d P_{2}}{d E}\right]
$$
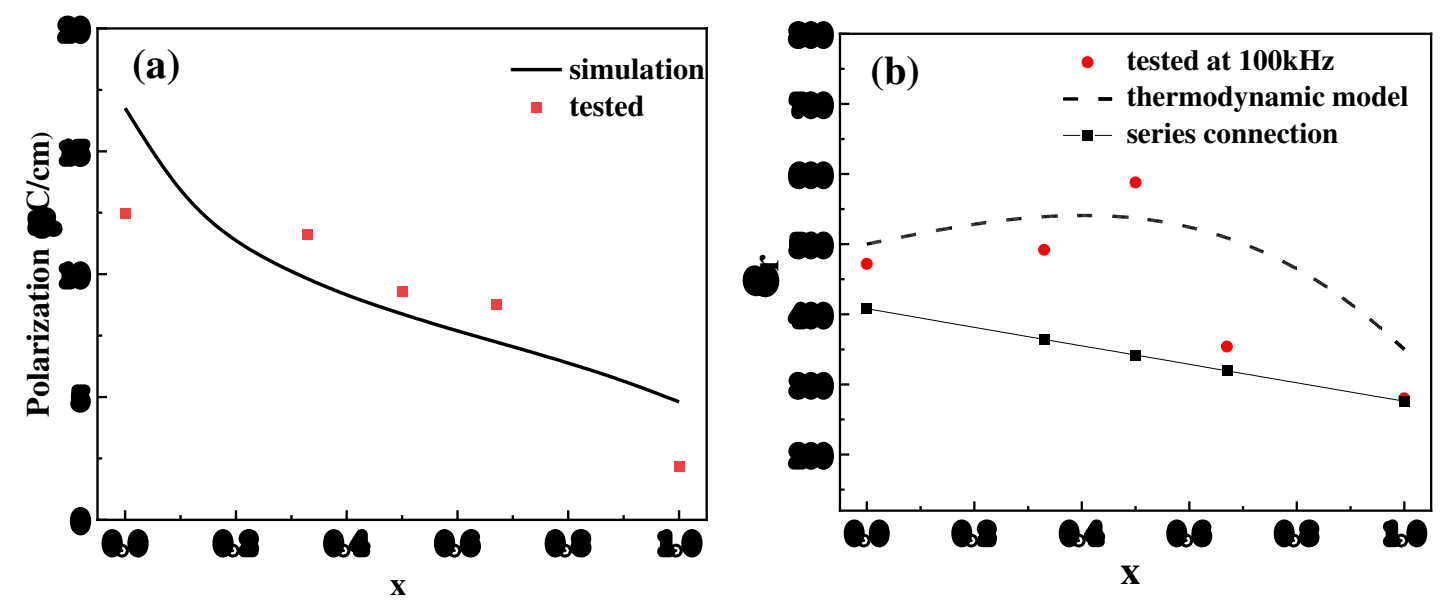

Fig.7 Mesoscopic Modeling: the polarization response of the tested and simulation result as a function of $x$ for PZT/BTO heterogeneous films(a), and the tested and thermodynamic analyzed dielectric constant as a function of $x$ for PZT/BTO heterogeneous films (b)

The corresponding polarization and dielectric responses of PZT/BTO heterogeneous film are shown in Fig.7 The polarization is calculated by the analytical solutions [32] of $\frac{\partial G}{\partial P_{1}}=0$ and $\frac{\partial G}{\partial P_{2}}=0$, which is basically consistent with the data obtained from the test when the external field $\mathrm{E}=0$ in Fig.7(a). According to the thermodynamic model, the dielectric constant of the material has a maximum value around $x=0.4$. According to the series connection capacitance theory, the dielectric constant was calculated and shown in Fig.7(b). The actual measured values fit the simulation curve when $x$ is close to 0.5 , and when $x$ increases, they further fit the curve from the series connection more closely. It indicated that when the content of a single layer is far more than the other one in the PZT/BTO heterogeneous films, the electrostatic coupling effect between the layers can be approximately negligible, which can be approximated as a series connection structure. Otherwise, in PZT/BTO heterogeneous films, the interlayer electrostatic coupling plays a great role, especially in the case of thickness ratio of $x=0.5$.

\section{Conclusions}

The electrostatically driven dielectric enhancement behaviors of PZT/BTO multilayer thin films with varying layer fractions onto $\mathrm{Pt} / \mathrm{Ti} / \mathrm{SiO}_{2} / \mathrm{Si}$ substrate were investigated. Better crystallinity and surface topography were found with heterogeneous structure. The results show that there exist a dielectric and ferroelectric enhancement when the BTO layer fraction $x$ is around 0.5 , the maximum of dielectric constant is 590 and the loss tangent is 0.14 at $100 \mathrm{kHz}$. We attribute this to the electrostatic fields result from the polarization and polarizability mismatch between the layers, and there exist a critical layer fraction of 0.5 , where the dielectric constant has the maximum value. For a miniature integrated capacitor with limited dimensions, this study enables the capacitor to have more storage capability with simple 
methods.

Acknowledgements This work was financially supported by National Natural Science Foundation of China (U1601208 and 51577070), the Key-Area Research and Development Program of Guangdong Province(2019B010937001, 2019B040403004, 2019B040403006), Natural Science Foundation of Guangdong Province (2019A1515012129), and Science and Technology Planning Project of Guangzhou City (202002030420). 


\section{References}

[1] A.R. Ramesh, S. Aggarwal, O. Auciello. Mater. Sci. Eng. R. Rep. 32, 191 (2001)

[2] N.M. Shorrocks, A. Patel, M. J. Walker et al., Microelectron. Eng.29, 59 (1995)

[3] A. K. Tagantsev, V. O. Sherman, K. F. Astafiev et al., J. Electroceram. 11, 5 (2003)

[4] A. Kumar, M. Kumar, V. Bhatt et al., Chem. Phys. Lett, 763,138162 (2020).

[5] A. Erbil ,Y. Kim , R. A. Gerhardt. Phys. Rev. Lett. 77, 1628 (1996)

[6]Kanno, Isaku, Jpn.J.Appl.Phys. 57, 040101 (2018)

[7] A. Kumar, S. Mukherjee, S. Sahare et al., Mat. Sci. Semicon. Proc, 122,105471 (2021).

[8] A. Kumar, D. Pednekar, S. Mukherjee et al., J. Mater Sci-Mater El, 27, 17055 (2020).

[9] D. Schwenk, F. Fishman, and F. Schwabl, J. Phys.: Condens. Matter. 2, 5409 (1990)

[10] J. Shen and Y. Q. Ma, Phys. Rev. B. 61, 14279 (2000)

[11] Q. L. Chen, Ann, Rev. Mater. Res. 32, 113 (2002)

[12] H. X. Cao, Z. Y. Li, Phys. Lett. A. 335, 444 (2005)

[13] Y. Li, W. Zhang, X. Wang et al., J. Porous. Mat. 15, 133 (2008)

[14] H. X. Cao, Z. Y. Li, Phys. Lett. A. 335, 444 (2005).

[15] R.G. Li, S.W. Jiang, L. B. Gao et al., Mater. Sci. Eng. B, 178, 911 (2013)

[16] J. Zhou, R. R. Li, Ru Li et al., Appl. Surf. Sci. 259, 29 (2012)

[17] H. Khassaf, N Khakpash, Vijayan S et al., Acta. Mater. 105, 68 (2016)

[18] M. T. Kesim, J. Zhang, S. P. Alpay et al., Appl. Phys. Lett. 105, 52901 (2014)

[19] M. B. Okatan, J. V. Mantese, S. P. Alpay, Phys. Rev. B. 79, 11 (2009)

[20] J. Zhang, M. W. Cole, S. P. Alpay, J. Appl. Phys. 108, 054103 (2010)

[21] A. L. Roytburd, S. Zhong, S. P. Alpay, Appl. Phys. Lett. 87, 092902 (2005)

[22] S. P. Alpay, J. Mantese, S. Trolier-Mckinstry et al., Mrs Bulletin, 39, 1099 (2014)

[23] H Liu, X Gong, J E Liang et al., Appl. Phys. Lett. 91, 122906 (2007).

[24] J. Zhu, Y. R. Li, Y. Zhang et al., Ceram. Int, 34, 967 (2008).

[25] N.D. Huang, Z.R Liu, Z. Q. W et al., Phys. Rev. Lett. 91, 602 (2003)

[26] J. Zhou, R. R. Li, Ru Li et al., Appl. Surf. Sci. 259, 29 (2012)

[27] D. Oneill, R. M. Bowman, J. M. Gregg, Appl. Phys. Lett. 77, 1520 (2000) 
[28] G. Catalan, D. Oneill, R. M. Bowman et al., Appl. Phys. Lett. 77, 3078 (2000)

[29] A. Sharma, Z. G. Ban, S. P. Alpay et al., Appl. Phys. Lett. 85, 985 (2004).

[30] N. A. Pertsev, A. G. Zembilgotov, A. K. Tagantsev, Phys. Rev. Lett. 80, 1988 (1998)

[31] M. J. Huang, E. Furman, H. A. MiKinstry et al., Ferroelectrics. 94, 313 (1989)

[32] A. L. Roytburd, S. Zhong, S.P. Alpay, Appl. Phys. Lett. 87, 092902 (2005) 